\title{
Genetical Analysis for Substiential Traits in New Yellow Maize Crosses Using Line X Tester Model
}

Neveen M. Hamouda ${ }^{1}$, A.A. El-Hosary ${ }^{1}$, S.A. Sedhom ${ }^{1}$, G.Y. Hamam ${ }^{1}$, T.A.E Saafan ${ }^{2}$ and A.A.A. El-Hosary ${ }^{1}$

1-Department of Agronomy, Faculty of Agriculture, Benha University, Egypt

2- Maize research dept., Field crops Inst. Agric. Res. Center.

\begin{abstract}
Sixteen yellow maize inbred lines were crossed to three testers of maize to estimate combining ability, for plant height, ear height, earliness and yield traits. The produce 48 crosses, SC Hytech 2088, SC Pioneer 3084, SC 3444, TWC Fine seeds 654 and TWC 352 were evaluated at the Farm of Faculty of Agriculture, Moshtohor, Benha University in RCBD with 3 replicates. Mean squares due to crosses (C), testers (T), inbred lines (L) and line $\mathrm{x}$ tester $(\mathrm{LxT})$ were significant $(\mathrm{P}<0.05)$ for all studied traits. Non- additive gene action $(\delta 2 \mathrm{SCA})$ is more pervading in determining inheritance of all studied traits. The contribution of inbred lines $\mathrm{x}$ testers to total variances as much higher than inbred lines or testers in all traits. However, the contribution of inbred lines to total variances was much higher than testers in all traits. The inbred line No 11 showed desirable (gii) for earliness traits and the best desirable combiner for grain yield / plant was No 3 followed by inbred line 11 then inbred line No1 and 10 since they expressed the highest significant and positive ( $\hat{g} i)$ effects.

. The cross L3xT3 exhibited Significant and negative Sij effects for days to 50\% tasseling and silking. The most desirable SCA effects for grain yield/ plant were detected by the top crosses combinations between T1 and each of L1,L2,L4,L7,L9, L10,L11, L13 and L16 ,also, between T2 and each of L3,L6, L8, L9,L10,L12,L15 and L16,also between T3 and each of L4,L5,L8,L12 and L14.
\end{abstract}

Key words: Combining ability, GCA, Inbred lines, SCA, Testers and Yellow Maize.

\section{Introduction}

Improved corn crosses need precise evaluation of hybrids under selection. Inbred lines performance per se does not extend an entirely suitable measure of their value in the line $x$ tester crosses. Thus, development of simple but effective method of evaluating new inbred lines and testers has been a major consideration in the development of new crosses. Line $\mathrm{x}$ tester technic has become a standard schema for evaluating both types of combining ability (GCA and SCA) of parental inbred lines to be used in hybrid combinations.

The line $\mathrm{x}$ tester schem with using different tester's base (narrow and broad) is the most prevalent method for the evaluating process. A wished tester described as give ultimate output on the predictable performance from the tested lines. Also, it combines the more simplicity utilization when used in other crosses or grown in various environments. No unique tester is able to completely fulfill these purposes (Sowmya et al. 2018, Arunkumar, et al. 2020 and ElHosary 2020).

There were unsolved problem that, chose the kind base of testers used in line $\mathrm{x}$ tester schema for assess inbred lines is still confused. Therefore, the choice of desirable tester is a serious decision. Utilization of low yielding variety carrying recessive factors for traits of economic interest should be used as a tester parent. But, the masking dominat desirable all else effect in such testers is making them ineffective. While, use of high yielding single crosses or elite inbred lines is useful for produce new three way crosses and single crosses, respectively. Also, assess of the top crosses gave a better idea SCA of the inbred lines. Matzinger (1953) reported that a narrow tester's genetic base participate more to line $\mathrm{x}$ tester interaction than does a large bases one. On the other hand, Grogan and Zuber (1957) illustrated that some double crosses like single crosses in effective for measuring GCA. El-Hosary (2014) estimated the relative value of various testers and found the small bases teeter like inbred line or single cross is more important in evaluating inbred line than open pollinated population. Sprague (1939) mentions that early testing for efficient test of inbred lines depend on bases of testers needed.

The ultimate goal of this study were to give an insight in the choice of desirable tester for evaluating inbred lines and determined superior inbred lines of maize, estimate GCA and SCA of the testers and inbred line.

\section{Materials and Methods}

Sixteen yellow maize inbred lines were top crossed to each of three various testers i.e. Inbred line L921, Population Iy-236 and SC Gold 21 (all materials were produce by family techno seed company except Population Iy-236 were produce from agriculture research center Egypt). The obtained 48 top crosses as well as five check hybrids were evaluated in summer 2019 in RCBD with three replication the five check hybrids were, SC Hytech 2088, SC Pioneer 3084, SC 3444, TWC Fine seeds 654 and TWC 352. Plot size was two ridges of $5 \mathrm{~m}$ length and $70 \mathrm{~cm}$ width. Each hill was spaced $25 \mathrm{~cm}$ apart with two kernels planted per hill and latter 
thinned to one plant per hill. Dray method of planting was used. The first irrigation was given after 21 days. The other irrigation was 10-15 days. The other cultural practices for maize production were applied as recommended. Data were recorded for days to $50 \%$ pollen shedding (tasseling date), days to $50 \%$ (silking date), plant height $(\mathrm{cm})$, ear height $(\mathrm{cm})$, No of rows/ear, No of kernels/ row, 100-kernel weight, ear weight $(\mathrm{g})$, grain yield/ plant $(\mathrm{g})$, shelling\%. Analysis of variance was performed in each trait according to kempthorne (1957) was followed to obtain information about the combining ability of different lines and testers and to determine different types of gene effects controlling the inheritance of traits under study.

\section{Results and Discussion}

Analysis of variance:

Analysis of variance of all studied traits is presented in Table (1). Significant mean squares due to top crosses along with lines, testers and line $\mathrm{x}$ testers were detected for all studied traits except, inbred lines for shelling\%, which was insignificant trait. Such results indicated a wide range of variability among parental inbred lines and testers. It is clear that the mean squares due to inbred lines were much higher than those of tester and line $\mathrm{x}$ tester for all traits except for shelling\%. Also, mean squares for inbred lines $\mathrm{x}$ testers were much higher than those of testers for plant height, 100-kernel weight, weight of ears and grain yield / plant and shelling\%. However, the other traits (tasselling date, silking date, ear height, number of rows/ ear and No. of kernels / row) mean squares for tester were much higher than those of inbred line $\mathrm{x}$ testers.

These results are in harmony with those obtained by EL-Hosary and EL-Gammaal (2013), Bayoumi (2018), El-Hosary (2020) and Ismail et al (2020). However, Amer and El-Shenawy (2007) reported that significant interactions $(\mathrm{P}<0.01)$ between treatments, lines $(\mathrm{L})$ and testers $(\mathrm{t})$ for earliness and grain yield plant-1. El-Morshidy et al. (2003) reported that lines were stable much more by change in environment than tester.

The estimation of variance due to general combining ability $\sigma 2 \mathrm{GCA}$ and specific combining ability $\sigma 2 \mathrm{SCA}$ are presented in Table (1). Results indicated that $\sigma 2 \mathrm{SCA}$ was more important than o2GCA for all studied traits. This is in harmony with the findings of several investigators who reported that $\sigma 2 \mathrm{SCA}$ is more sensitive to environmental changes than 62 GCA (El-Hosary 2020 and Ismail et al (2020).

The contribution of inbred lines, testers and their interactions to total variance are presented in Table (1). The contribution of inbred lines $\mathrm{x}$ testers to total variances as much higher than inbred lines or testers in all traits. However, the contribution of inbred lines to total variances was much higher than testers in all traits. These results are in agreement with obtained by Darshan and Marker (2019) and Gamea (2019). In the same context, Mbuvi et al, (2018) used thirty inbred lines and two testers in a line by tester analysis to evaluate the GCA and SCA effects for yield and associated traits. The analysis showed highly significant GCA and SCA indicating that both additive and non-additive gene effects contributed to the inheritance of the traits studied. Sum of squares of GCA was more than of SCA hence additive main effects contributed more to the inheritance of the traits than non-additive gene effects.

Table 1. Observed mean squares from ordinary analysis and combining ability for the studied traits.

\begin{tabular}{|c|c|c|c|c|c|c|c|c|c|c|}
\hline SO V & d.f & $\begin{array}{c}\text { Days to } \\
50 \% \\
\text { shed }\end{array}$ & $\begin{array}{c}\text { days to } \\
50 \% \\
\text { silk }\end{array}$ & $\begin{array}{l}\text { Plant } \\
\text { height } \\
(\mathrm{cm})\end{array}$ & $\begin{array}{l}\text { Ear height } \\
\quad(\mathrm{cm})\end{array}$ & $\begin{array}{c}\text { Number } \\
\text { of } \\
\text { rows/ } \\
\text { ear } \\
\end{array}$ & $\begin{array}{c}100- \\
\text { kernel } \\
\text { weight } \\
(\mathrm{g})\end{array}$ & $\begin{array}{l}\text { ear weight } \\
\text { / plant (g) }\end{array}$ & $\begin{array}{c}\text { grain } \\
\text { yield/ } \\
\text { plant (g) }\end{array}$ & shelling $\%$ \\
\hline Reps & 2 & $9.63 * *$ & $17.42 * *$ & $886.31 * *$ & $1021.41 * *$ & $10 . .90$ & $41.30^{*}$ & 0.59 & 3.73 & 0.01 \\
\hline Crosses & 47 & $3.96 * *$ & $3.76^{* *}$ & $551.68^{* *} *$ & $386.98 * *$ & $19.16^{* * *}$ & $36.26^{* *}$ & $1608.44 * *$ & $1110.24 * *$ & $0.02 * *$ \\
\hline Lines & 15 & $19.59^{* *}$ & $17.69 * *$ & $2804.21^{* *}$ & $2394.77 * *$ & 56. $27 * *$ & $129.78^{* *} *$ & $5343.85^{* *}$ & $3920.50 * *$ & 0.0007 \\
\hline Testers & 2 & $3.92 * *$ & $3.38 * *$ & $446.11^{* *}$ & $346.62 * *$ & $21.08 * *$ & $23.61 *$ & $1229.93 * *$ & $753.36 * *$ & 0.0017 \\
\hline Line $\mathrm{x}$ Tester & 30 & $2.93 * *$ & $3.02 * *$ & $454.29 * *$ & $273.31 * *$ & $15.73 * *$ & $36.36 * *$ & $1548.67 * *$ & $1101.33 * *$ & 0.0019 \\
\hline Eror & 94 & 1.11 & 1.44 & 153.33 & $65 . .30$ & 5. .34 & 11.23 & 0.69 & 1.46 & 0.0002 \\
\hline$\sigma 2 \mathrm{GCA}$ & & 0.01 & 0.01 & 1.17 & 1.36 & 0.04 & 0.00 & 0.72 & 0.11 & -0.000005 \\
\hline$\sigma 2 \mathrm{SCA}$ & & 0.02 & 0.02 & 2.33 & 2.72 & 0.08 & 0.00 & 1.43 & 0.21 & -0.00001 \\
\hline $\begin{array}{c}\text { CONTRIBUTION } \\
\text { OF LINES }\end{array}$ & & 31.65 & 28.68 & 25.81 & 28.59 & 35.11 & 20.78 & 24.40 & 21.66 & 15.36 \\
\hline $\begin{array}{c}\text { CONTRIBUTION } \\
\text { OF TESTERS }\end{array}$ & & 21.07 & 20.03 & 21.63 & 26.33 & 12.50 & 15.23 & 14.14 & 15.03 & 4.66 \\
\hline $\begin{array}{c}\text { CONTRIBUTION } \\
\text { OF (L X T) }\end{array}$ & & 47.28 & 51.29 & 52.56 & 45.08 & 52.40 & 63.99 & 61.46 & 63.32 & 79.98 \\
\hline
\end{tabular}

$*$ and $* *$ refers to significant $\mathrm{p}>0.05$ and $\mathrm{p}>0.01$, respectively. 


\section{Mean performance}

Mean performance of crosses as well as check hybrids for tasseling and silking dates, plant and ear heights, ear leaf area, ear length, No. of kernels/ row , No of rows/ ear, 100-kernel weight, ear weight/ plants, grain yield / plant and shelling\% are presented in Table (2) .

For days to tasseling, the three way cross (TWC) L3xT3 (Gold21) expressed significant earliest compared with the best check variety single cross Pioneer SC 3444. However, the top crosses L3xT1 (L921), T2 (Pop. Iy-236) with each of lines No. 5, 8, 12 and 13 and all top crosses between T3 (Gold21) and all lines expressed insignificant compared with the best check single cross Pioneer 3444. For silking date, the top cross L3xT3 (Gold21) exhibited significantly earliest than the best check (SC 3444) for this trait. However, the other top crosses gave insignificant for this trait except for crosses i.e. T2 and each of L5,L8,L12 and L13 .

From the previous results it could be concluded that the inbred line No.3 and tester 3 (Gold21) and their top crosses could be utilized developing early flowering hybrid since they were the earliest among top crosses.

Regarding plant height, all top crosses were significant higher than TWC 654 except the top cross L12xT1. However, the top crosses between T1 and each of lines No.3,7,10 and 13, and T2 and each of inbred lines No.,5,8,11,12,14,15and16, and T3 and each of inbred lines No.1,2,3,7,8,10,14 and 16 exhibited insignificant of highest plant hight compare with the highest check hybrid SC Pioneer 3084 . It could be concluded that the previous top crosses could be utilized for silage production.

For ear height, the top crosses L12xT1 and L1xT1 expressed significant lowest ear high compare with the best check hybrid TWC 352. Also, all top crosses showed non-significant lowest values as compared with the best check TWC 352 except nine top crosses i.e. L10xT1, L4xT2, L5xT2, L8xT2, L14xT2 , L10xT3, L11xT3, L12xT3 and L14xT3 where exhibited significantly higher value of this trait.

As for number of kernels/row, fifteen top crosses had insignificant differences compare the best check SC Hytech 2088. The top cross L4xT2 gave the highest No of kernels /row.

For No. of rows/ ear, two, three and five top crosses exhibited significantly relative the best SC Pioneer 3084 and 3444. However, the top cross T2xL9 gave significantly higher number of rows/ ear compare with TWC352.

For 100-kernel weight, twelve, seven, and six top crosses exhibited insignificantly relative the best check hybrid Hytech 2088. The best top crosses L7xT1, L9xT1, L5xT3 and L14xT3, also, all three way crosses (T3 and each of all inbred lines) gave significant higher or insignificant compare to TWC 654. The best top crosses L4xT3 and L1xT3 had significantly heavier than TWC 352.

For ear weight/ plant, all top crosses showed significantly lowest compare with check hybrids (Pioneer.3084 and SC 3444). While, the top cross L7xT1 showed significantly higher than check variety Hytech2088). However, the top crosses L1xT1, L4xT1, L10xT1, L11xT1, L3xT3, L5xT3 and L8xT3 exhibited insignificant compare the check variety Hytech 2088. Also, the top crosses (TWC) L1xT3, L4xT3, L8xT3, L11xT3 and L14xT3 expressed significantly higher compare the best check variety TWC 654.

Grain yield / plant, all top crosses exhibited significantly lower than check variety SC Pioneer 3084 or SC 3444.

The top crosses between T1 and each of L1, L4, L7,L10, L11 and L5xT3 showed significantly surpassed hybrid SC Hytech2088. The top crosses L3xT1, L15xT1, L16xT1, L3xT2, L3xT3, L4xT3, L8xT3, L11xT3 and L14xT3 showed insignificant differed from SC Pioneer 2088. However, the top cross (TWC) obtained between T3 (Gold21) and each of inbred lines L1, L3, L4, L5, L8, L11 and L14 exhibited significant highest yield compare with best T WC Fine seeds 654.

From such results it could be concluded that the previous top crosses could be efficient and prospective in maize breeding programs since they expressed significant desirable effects for grain yield and for one or more of yield components.

\section{Analysis of variance for combining ability:}

The estimation of variance due to general combining ability ( $\sigma 2 \mathrm{GCA})$ and specific combining ability ( $\sigma 2 \mathrm{SCA}$ ) are presented in Table (1) .Results indicated that $(\sigma 2 \mathrm{SCA})$ was more important than $(\sigma 2 \mathrm{GCA})$ for all studied traits . such results are in agreement with those reported by Sadek et al. (2002) and Ahmed et al. (2017).

General Combining ability effect:

The general combining ability effects (ĝi) of testers and parental inbred lines for all traits are presented in Table (3). From the breeder's point of view, high negative values of tassling and silking date as well as plant and ear heights along with high positive values for yield and its components would be useful for maize breeding program .

Inbred lines:

The parental inbred lines No.11, 9 and 4 expressed desirable significant (ĝi) effects for tasseling date. While, the most desirable combiner for this trait was inbred line No 11. Also, the parental inbred line No.11 gave the desirable (ĝi) effect for silking date. From these inbred lines, line No11 was common for both traits. The best inbred lines in general combining ability effects (ĝi) were No. 12, 15 and 7 for ear height and L12 for plant height. This inbred line expressed significant negative (ĝi) effects for both traits. From these inbred lines, inbred line no 12 was common for both traits. 
Table 2. Mean performance of the top crosses and check varieties.

\begin{tabular}{|c|c|c|c|c|c|c|c|c|c|}
\hline Trait & \multicolumn{3}{|c|}{$\begin{array}{c}\text { Tasseling date } \\
\text { ( Days to } 50 \% \text { shed) }\end{array}$} & \multicolumn{3}{|c|}{$\begin{array}{c}\text { Silking date } \\
\text { (Days to } 50 \% \text { silk) }\end{array}$} & \multicolumn{3}{|c|}{ Plant height $(\mathrm{cm})$} \\
\hline Tester & $\begin{array}{c}\mathrm{T} 1 \\
\text { In bred } \\
\text { line } \mathrm{I} .921\end{array}$ & $\begin{array}{c}\text { T2 } \\
\text { Pop.Iy } \\
-236\end{array}$ & $\begin{array}{c}\text { T3 } \\
\text { Gold } \\
21\end{array}$ & $\begin{array}{c}\mathrm{T} 1 \\
\text { In bred } \\
\text { line } \mathrm{I} .921\end{array}$ & $\begin{array}{c}\text { T2 } \\
\text { Pop.Iy- } \\
236\end{array}$ & $\begin{array}{c}\text { T3 } \\
\text { Gold } \\
21\end{array}$ & $\begin{array}{c}\text { T1 } \\
\text { In } \\
\text { bred } \\
\text { line } \\
\text { I.921 } \\
\end{array}$ & $\begin{array}{c}\text { T2 } \\
\text { Pop.Iy } \\
-236\end{array}$ & $\begin{array}{c}\text { T3 } \\
\text { Gold } \\
21\end{array}$ \\
\hline 1 & 58.67 & 57.33 & $\begin{array}{c}58.0 \\
0\end{array}$ & 61.67 & 60.33 & 61.00 & $\begin{array}{c}250.5 \\
7\end{array}$ & $\begin{array}{c}251.1 \\
0\end{array}$ & $\begin{array}{c}273.8 \\
7\end{array}$ \\
\hline 2 & 57.00 & 58.00 & $\begin{array}{c}57.6 \\
7\end{array}$ & 60.00 & 61.00 & 60.33 & $\begin{array}{c}254.4 \\
0\end{array}$ & $\begin{array}{c}237.1 \\
0\end{array}$ & $\begin{array}{c}266.6 \\
3\end{array}$ \\
\hline 3 & 59.33 & 58.67 & $\begin{array}{c}55.0 \\
0\end{array}$ & 62.33 & 61.33 & 58.00 & $\begin{array}{c}268.3 \\
3\end{array}$ & $\begin{array}{c}254.4 \\
0\end{array}$ & $\begin{array}{c}272.6 \\
3\end{array}$ \\
\hline 4 & 57.67 & 57.67 & $\begin{array}{c}56.3 \\
3\end{array}$ & 60.67 & 60.33 & 59.33 & $\begin{array}{c}243.8 \\
7\end{array}$ & $\begin{array}{c}262.7 \\
3\end{array}$ & $\begin{array}{c}257.7 \\
7\end{array}$ \\
\hline 5 & 58.00 & 60.67 & $\begin{array}{c}58.3 \\
3\end{array}$ & 60.33 & 63.67 & 61.67 & $\begin{array}{c}260.5 \\
3\end{array}$ & $\begin{array}{c}285.5 \\
3\end{array}$ & $\begin{array}{c}260.5 \\
3\end{array}$ \\
\hline 6 & 58.00 & 58.67 & $\begin{array}{c}59.0 \\
0\end{array}$ & 61.00 & 61.33 & 62.00 & $\begin{array}{c}248.8 \\
3\end{array}$ & $\begin{array}{c}258.3 \\
0\end{array}$ & $\begin{array}{c}274.9 \\
7\end{array}$ \\
\hline 7 & 57.33 & 57.00 & $\begin{array}{c}58.0 \\
0\end{array}$ & 60.67 & 60.00 & 61.00 & $\begin{array}{c}263.3 \\
3\end{array}$ & $\begin{array}{c}239.4 \\
0\end{array}$ & $\begin{array}{c}265.5 \\
0\end{array}$ \\
\hline 8 & 58.00 & 60.67 & $\begin{array}{c}59.0 \\
0\end{array}$ & 61.00 & 63.33 & 61.67 & $\begin{array}{c}257.7 \\
7\end{array}$ & $\begin{array}{c}273.3 \\
0\end{array}$ & $\begin{array}{c}287.7 \\
3\end{array}$ \\
\hline 9 & 56.00 & 59.00 & $\begin{array}{c}56.0 \\
0\end{array}$ & 59.33 & 62.00 & 59.00 & $\begin{array}{c}246.0 \\
7\end{array}$ & $\begin{array}{c}251.1 \\
0\end{array}$ & $\begin{array}{c}259.9 \\
3\end{array}$ \\
\hline 10 & 57.33 & 59.00 & $\begin{array}{c}58.0 \\
0\end{array}$ & 60.67 & 61.67 & 60.33 & $\begin{array}{c}272.1 \\
7\end{array}$ & $\begin{array}{c}254.9 \\
7\end{array}$ & $\begin{array}{c}273.2 \\
7\end{array}$ \\
\hline 11 & 55.33 & 58.33 & $\begin{array}{c}57.0 \\
0\end{array}$ & 58.33 & 61.67 & 59.67 & $\begin{array}{c}253.3 \\
3\end{array}$ & $\begin{array}{c}274.4 \\
0\end{array}$ & $\begin{array}{c}255.6 \\
0\end{array}$ \\
\hline 12 & 57.67 & 60.00 & $\begin{array}{c}58.8 \\
3\end{array}$ & 60.67 & 62.67 & 61.67 & $\begin{array}{c}221.1 \\
0\end{array}$ & $\begin{array}{c}265.5 \\
0\end{array}$ & $\begin{array}{c}243.3 \\
0\end{array}$ \\
\hline 13 & 57.33 & 59.33 & $\begin{array}{c}58.6 \\
7\end{array}$ & 60.00 & 62.33 & 61.67 & $\begin{array}{c}262.2 \\
0\end{array}$ & $\begin{array}{c}257.7 \\
3\end{array}$ & $\begin{array}{c}254.4 \\
0\end{array}$ \\
\hline 14 & 57.67 & 58.00 & $\begin{array}{c}57.6 \\
7\end{array}$ & 60.33 & 60.67 & 60.33 & $\begin{array}{c}228.8 \\
7\end{array}$ & $\begin{array}{c}262.2 \\
0\end{array}$ & $\begin{array}{c}271.1 \\
7\end{array}$ \\
\hline 15 & 58.00 & 58.00 & $\begin{array}{c}57.3 \\
3\end{array}$ & 61.00 & 61.00 & 60.00 & $\begin{array}{c}244.8 \\
7\end{array}$ & $\begin{array}{c}274.4 \\
3\end{array}$ & $\begin{array}{c}260.5 \\
3\end{array}$ \\
\hline 16 & 58.33 & 58.33 & $\begin{array}{c}57.3 \\
3\end{array}$ & $\begin{array}{r}61.00 \\
\text { Checks }\end{array}$ & 61.00 & 60.33 & $\begin{array}{c}253.8 \\
3\end{array}$ & $\begin{array}{c}278.8 \\
7\end{array}$ & $\begin{array}{c}277.8 \\
7\end{array}$ \\
\hline $\begin{array}{l}1 \text { ( SC } 2088 \\
\text { Hytech) }\end{array}$ & & 57.67 & & & 60.67 & & & 282.20 & \\
\hline $\begin{array}{l}2 \text { (SC } 3084 \\
\text { pioneer) }\end{array}$ & & 59.00 & & & 62.00 & & & 285.53 & \\
\hline $\begin{array}{l}3 \text { (SC } 3444 \\
\text { pioneer) }\end{array}$ & & 57.33 & & & 60.33 & & & 279.40 & \\
\hline $\begin{array}{l}4 \text { (TWC } 654 \text { fain } \\
\text { seeds ) }\end{array}$ & & 59.33 & & & 62.33 & & & 252.17 & \\
\hline 5 ( TWC 352) & & 57.67 & & & 61.00 & & & 251.83 & \\
\hline LSD 0.05 & & 1.71 & & & 1.94 & & & 20.07 & \\
\hline LSD 0.01 & & 2.26 & & & 2.57 & & & 26.58 & \\
\hline
\end{tabular}




\begin{tabular}{|c|c|c|c|c|c|c|c|c|c|}
\hline Trait & \multicolumn{3}{|c|}{ Ear height $(\mathrm{cm})$} & \multicolumn{3}{|c|}{ Number of rows/ ear } & \multicolumn{3}{|c|}{$\begin{array}{c}\text { Number of } \\
\text { kernels/ row }\end{array}$} \\
\hline $\begin{array}{l}\text { Tester } \\
\text { Line }\end{array}$ & $\begin{array}{c}\mathrm{T} 1 \\
\text { In bred line } \\
\mathrm{I} .921\end{array}$ & $\begin{array}{c}\text { T2 } \\
\text { Pop.Iy- } \\
236\end{array}$ & $\begin{array}{c}\text { T3 } \\
\text { Gold } \\
21\end{array}$ & $\begin{array}{c}\mathrm{T} 1 \\
\text { In bred line } \\
\mathrm{I.921}\end{array}$ & $\begin{array}{c}\text { T2 } \\
\text { Pop.Iy- } \\
236\end{array}$ & $\begin{array}{c}\text { T3 } \\
\text { Gold } \\
21\end{array}$ & $\begin{array}{c}\mathrm{T} 1 \\
\mathrm{In} \\
\text { bre } \\
\mathrm{d} \\
\text { line } \\
\mathrm{I} .92 \\
1\end{array}$ & $\begin{array}{c}\text { T2 } \\
\text { Pop.I } \\
\text { y- } \\
236\end{array}$ & $\begin{array}{c}\mathrm{T} 3 \\
\text { Gol } \\
\mathrm{d} \\
21\end{array}$ \\
\hline 1 & 116.63 & 116.63 & $\begin{array}{c}146.6 \\
3\end{array}$ & 14.00 & 16.00 & 16.67 & $\begin{array}{l}37 . \\
33\end{array}$ & $\begin{array}{c}34.3 \\
3\end{array}$ & $\begin{array}{l}38 . \\
00\end{array}$ \\
\hline 2 & 122.73 & 116.63 & $\begin{array}{c}138.3 \\
0\end{array}$ & 14.67 & 13.33 & 14.00 & $\begin{array}{r}34 . \\
67\end{array}$ & $\begin{array}{c}30.6 \\
7\end{array}$ & $\begin{array}{l}39 . \\
00\end{array}$ \\
\hline 3 & 133.87 & 125.53 & $\begin{array}{c}140.0 \\
0\end{array}$ & 16.67 & 14.00 & 14.00 & $\begin{array}{l}34 . \\
67\end{array}$ & $\begin{array}{c}40.0 \\
0\end{array}$ & $\begin{array}{r}40 . \\
00\end{array}$ \\
\hline 4 & 122.87 & 136.63 & $\begin{array}{c}146.0 \\
7\end{array}$ & 14.00 & 13.33 & 13.33 & $\begin{array}{l}37 . \\
00\end{array}$ & $\begin{array}{c}40.6 \\
7\end{array}$ & $\begin{array}{r}40 . \\
00\end{array}$ \\
\hline 5 & 126.63 & 152.73 & $\begin{array}{c}127.1 \\
7\end{array}$ & 13.33 & 16.00 & 14.00 & $\begin{array}{l}36 . \\
00\end{array}$ & $\begin{array}{c}34.0 \\
0\end{array}$ & $\begin{array}{r}40 . \\
00\end{array}$ \\
\hline 6 & 135.50 & 132.77 & $\begin{array}{c}138.3 \\
0\end{array}$ & 17.33 & 14.00 & 13.33 & $\begin{array}{l}34 . \\
67\end{array}$ & $\begin{array}{c}40.0 \\
0\end{array}$ & $\begin{array}{r}40 . \\
00\end{array}$ \\
\hline 7 & 120.53 & 127.10 & $\begin{array}{c}127.2 \\
0\end{array}$ & 14.00 & 14.00 & 16.00 & $\begin{array}{l}36 . \\
67\end{array}$ & $\begin{array}{c}32.0 \\
0\end{array}$ & $\begin{array}{l}38 . \\
33\end{array}$ \\
\hline 8 & 123.77 & 147.20 & $\begin{array}{c}152.1 \\
7\end{array}$ & 14.67 & 12.67 & 16.00 & $\begin{array}{l}36 . \\
67\end{array}$ & $\begin{array}{c}39.3 \\
3\end{array}$ & $\begin{array}{l}39 . \\
00\end{array}$ \\
\hline 9 & 121.07 & 135.00 & $\begin{array}{c}127.7 \\
7\end{array}$ & 13.33 & 18.00 & 13.33 & $\begin{array}{l}37 . \\
00\end{array}$ & $\begin{array}{c}35.3 \\
3\end{array}$ & $\begin{array}{l}36 . \\
67\end{array}$ \\
\hline 10 & 136.67 & 135.50 & $\begin{array}{c}139.4 \\
0\end{array}$ & 15.33 & 14.67 & 14.00 & $\begin{array}{l}36 . \\
33\end{array}$ & $\begin{array}{c}39.0 \\
0\end{array}$ & $\begin{array}{l}36 . \\
67\end{array}$ \\
\hline 11 & 118.30 & 129.97 & $\begin{array}{c}138.8 \\
7\end{array}$ & 14.00 & 13.33 & 16.00 & $\begin{array}{l}40 . \\
00\end{array}$ & $\begin{array}{c}40.3 \\
3\end{array}$ & $\begin{array}{l}38 . \\
00\end{array}$ \\
\hline 12 & 107.73 & 128.30 & $\begin{array}{c}118.0 \\
2\end{array}$ & 14.00 & 14.67 & 13.33 & $\begin{array}{l}32 . \\
00\end{array}$ & $\begin{array}{c}38.6 \\
7\end{array}$ & $\begin{array}{l}35 . \\
33\end{array}$ \\
\hline 13 & 122.20 & 136.10 & $\begin{array}{c}121.5 \\
3\end{array}$ & 15.33 & 14.00 & 16.00 & $\begin{array}{l}35 \\
67\end{array}$ & $\begin{array}{c}34.3 \\
3\end{array}$ & $\begin{array}{l}35 . \\
00\end{array}$ \\
\hline 14 & 107.73 & 154.40 & $\begin{array}{c}131.1 \\
0\end{array}$ & 14.00 & 14.00 & 13.33 & $\begin{array}{l}32 . \\
33\end{array}$ & $\begin{array}{c}34.3 \\
3\end{array}$ & $\begin{array}{l}36 . \\
33\end{array}$ \\
\hline 15 & 113.87 & 124.97 & $\begin{array}{c}126.6 \\
3\end{array}$ & 14.00 & 16.00 & 16.00 & $\begin{array}{c}38 . \\
67\end{array}$ & $\begin{array}{c}36.0 \\
0\end{array}$ & $\begin{array}{l}35 . \\
67\end{array}$ \\
\hline 16 & 126.07 & 149.97 & $\begin{array}{c}133.3 \\
0 \\
\end{array}$ & 14.00 & 13.33 & 12.00 & $\begin{array}{l}37 . \\
00 \\
\end{array}$ & $\begin{array}{c}34.0 \\
0 \\
\end{array}$ & $\begin{array}{l}39 . \\
33 \\
\end{array}$ \\
\hline & & & Che & & & & & & \\
\hline $\begin{array}{l}1 \text { ( SC } 2088 \\
\text { Hytech) }\end{array}$ & \multicolumn{3}{|c|}{136.67} & \multicolumn{3}{|c|}{12.00} & \multicolumn{3}{|c|}{42.33} \\
\hline $\begin{array}{l}2 \text { (SC } 3084 \\
\text { pioneer) }\end{array}$ & \multicolumn{3}{|c|}{146.07} & \multicolumn{3}{|c|}{14.00} & \multicolumn{3}{|c|}{40.00} \\
\hline $\begin{array}{l}3 \text { (SC } 3444 \\
\text { pioneer) }\end{array}$ & \multicolumn{3}{|c|}{131.63} & \multicolumn{3}{|c|}{14.00} & \multicolumn{3}{|c|}{40.00} \\
\hline $\begin{array}{l}4 \quad \text { (TWC } 654 \\
\text { fain seeds })\end{array}$ & \multicolumn{3}{|c|}{129.43} & \multicolumn{3}{|c|}{14.00} & \multicolumn{3}{|c|}{36.67} \\
\hline 5 ( TWC 352) & \multicolumn{3}{|c|}{123.30} & \multicolumn{3}{|c|}{16.00} & \multicolumn{3}{|c|}{36.33} \\
\hline LSD 0.05 & \multicolumn{3}{|c|}{13.10} & \multicolumn{3}{|c|}{1.76} & \multicolumn{3}{|c|}{3.74} \\
\hline LSD 0.01 & \multicolumn{3}{|c|}{17.35} & \multicolumn{3}{|c|}{12.00} & \multicolumn{3}{|c|}{4.96} \\
\hline
\end{tabular}




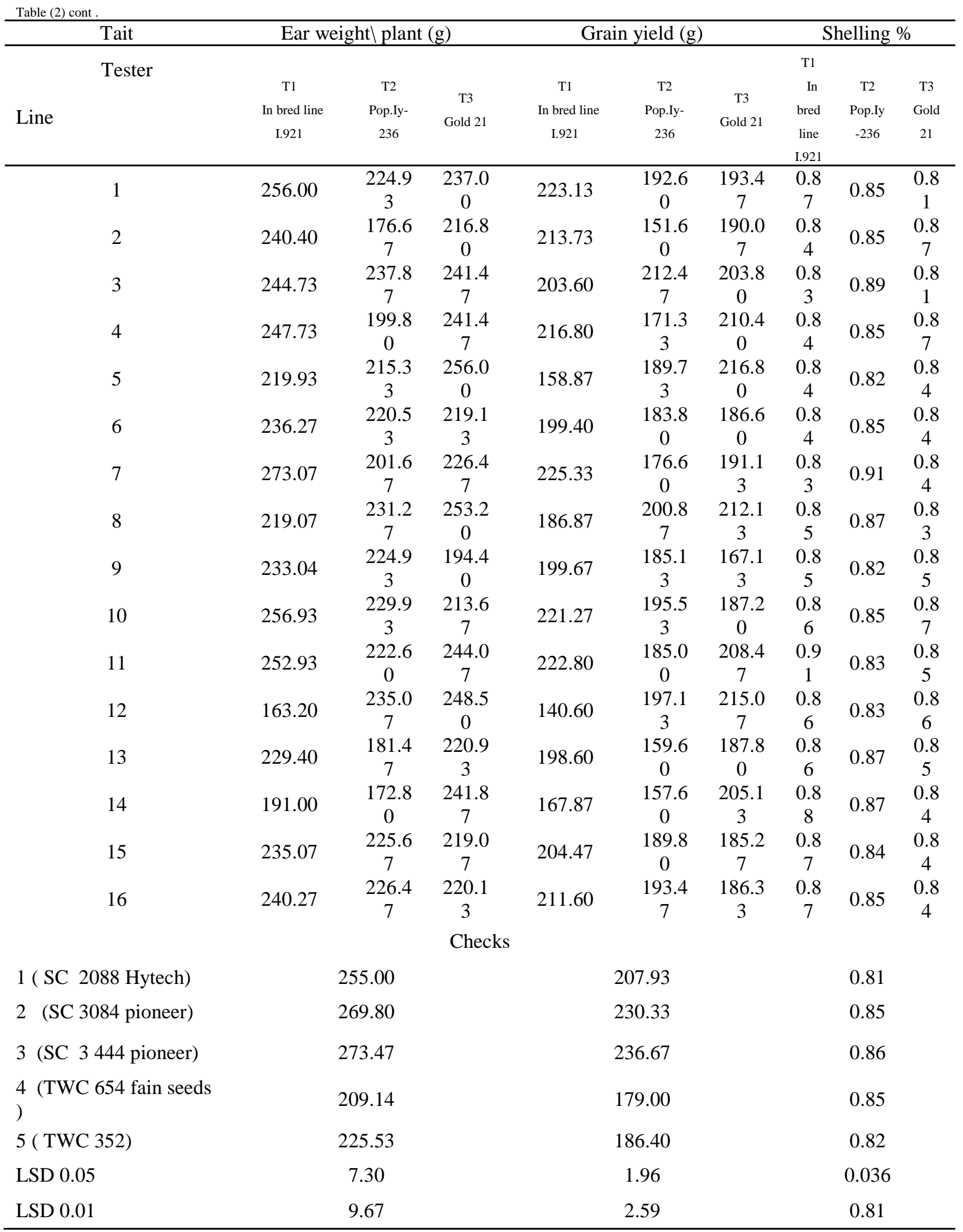

Therefore, they could be of great value in breeding programs for developing new hybrids with short plant stature. For No of kernels/ row No 1 and 15 for No of rows/ ear, No 7 and 16 for 100-kernel weight. These inbred lines expressed significant and positive (ĝi) effects for the previously mentioned traits would be utilized in corn breeding programs.
For ear weight, grain yield and shelling\%, the best general combining ability which had significant positive (ĝi) effects were No.1, 3, 4, 7,8,10 and 11 . Moreover, the best desirable combiner for grain yield / plant was No 3 followed by inbred line 11 then inbred line No1 and 10 since they expressed the highest significant and positive (ĝi) effects . The 
inbred lines No3, 4, 7 and 11 which had high ( $\hat{\mathrm{g} i})$ effect for grain yield was among the best combiners for one or more for yield components. Therefore, these inbred lines could be of great value in breeding programs for improving grain yield and its components.

Testers:

General combining ability effects for the three testers are presented in Table (3). The first tester inbred line 921 expressed significant desirable ( $\hat{\mathrm{g} i}$ ) effects for tasseling date, plant and ear heights , 100kernel weight, shelling\%, ear weight and grain yield/ plant . Parental tester population yellow 236 gave the best general combiner for tasseling and silking date, No. of kernels/row, ear and grain yield/ plant. From such results, it could be concluded that the first tester (inbred line 921) was the best tester for grain yield and its components. The superiority of inbred lines as good tester noticed by general workers Iqbal et al. (2007), Rajitha et al. (2014), Hosana et al. (2015), Abd El-Zaher (2016), Darshan and Marker (2019) and Gamea (2019).

The third tester (Gold 21) expressed significant desirable gi effects for silking date, No of kernels/ row, shelling\%, ear weight/ plant and grain yield/ plant.

The population yellow 236 had the best combiners for ear leaf area only. The superiority of population or upon variety as good tester noticed by several workers Ibrahim et al. (2005)., Osman and Ibrahim (2007) , Ahmed (2008) ., and Lai et al. (2011) which gave negative combiners for most traits.

Table 3. General combining ability effects of testers and inbred lines for all the studied traits .

\begin{tabular}{|c|c|c|c|c|c|}
\hline Trait & $\begin{array}{l}\text { Tasseling date } \\
\text { (Days to 50\% } \\
\text { shed) }\end{array}$ & $\begin{array}{l}\text { Silking date (Days } \\
\text { to } 50 \% \text { silk) }\end{array}$ & Plant height $(\mathrm{cm})$ & $\begin{array}{l}\text { Ear height } \\
\quad(\mathrm{cm})\end{array}$ & $\begin{array}{c}\text { Numb } \\
\text { er of } \\
\text { kernel } \\
\text { s /row }\end{array}$ \\
\hline \multicolumn{6}{|l|}{ Tester } \\
\hline 1 & $-0.33^{*}$ & -0.26 & $-8.19 * *$ & $-8.15 * *$ & $-0.81 *$ \\
\hline 2 & $0.74 * *$ & $0.69 * *$ & 1.25 & $3.92 * *$ & -0.42 \\
\hline 3 & $-0.41 * *$ & $-0.43^{*}$ & $6.94 * *$ & $4.23 * *$ & $1.23 * *$ \\
\hline LSD 0.05 & 0.30 & 0.34 & 3.55 & 2.32 & 0.66 \\
\hline LSD 0.01 & 0.40 & 0.46 & 4.70 & 3.07 & 0.88 \\
\hline \multicolumn{6}{|l|}{ Lines } \\
\hline 1 & 0.07 & 0.17 & -1.56 & -3.78 & -0.30 \\
\hline 2 & -0.38 & -0.38 & -7.36 & -4.53 & $\begin{array}{c}- \\
2.08 * *\end{array}$ \\
\hline 3 & -0.26 & -0.27 & 5.05 & 2.72 & 1.37 \\
\hline 4 & $-0.71 *$ & -0.72 & -5.28 & 4.77 & $2.37 * *$ \\
\hline 5 & $1.07 * *$ & $1.06 * *$ & $8.80 *$ & 5.10 & -0.19 \\
\hline 6 & 0.63 & 0.62 & 0.63 & 5.11 & 1.37 \\
\hline 7 & -0.49 & -0.27 & -3.99 & $-5.47 *$ & -1.19 \\
\hline 8 & $1.29 * *$ & $1.17 * *$ & $12.86^{* *}$ & $10.63 * *$ & 1.48 \\
\hline 9 & $-0.93 * *$ & -0.72 & -7.70 & -2.47 & -0.52 \\
\hline 10 & 0.18 & 0.06 & 6.73 & $6.77 *$ & 0.48 \\
\hline 11 & $-1.04 * *$ & $-0.94 *$ & 1.02 & -1.37 & $2.59 * *$ \\
\hline 12 & 0.29 & 0.28 & $-11.25 * *$ & $-11.78 * *$ & -0.85 \\
\hline 13 & 0.51 & 0.51 & -1.96 & -3.80 & $-1.85^{*}$ \\
\hline 14 & -0.15 & -0.38 & -5.99 & 0.66 & $\begin{array}{c}- \\
2.52 * *\end{array}$ \\
\hline 15 & -0.15 & -0.16 & -0.13 & $-8.59 * *$ & -0.08 \\
\hline 16 & 0.07 & -0.05 & $10.12^{*}$ & $6.03 *$ & -0.08 \\
\hline LSD 0.05 & 0.70 & 0.79 & 8.20 & 5.35 & 1.53 \\
\hline LSD 0.01 & 0.92 & 1.05 & 10.85 & 7.08 & 2.02 \\
\hline
\end{tabular}


Table (3) : Cont

\begin{tabular}{|c|c|c|c|c|c|}
\hline Trait & $\begin{array}{c}\text { Number of } \\
\text { rows } \backslash \text { ear }\end{array}$ & $\begin{array}{l}\text { 100-kernel } \\
\text { weight }(\mathrm{g})\end{array}$ & $\begin{array}{c}\text { Ear weight / } \\
\text { plant (g) }\end{array}$ & $\begin{array}{l}\text { Grain yield } \\
\text { /plant (g) }\end{array}$ & Shelling \% \\
\hline \multicolumn{6}{|l|}{ Tester } \\
\hline 1 & 0.06 & $1.89 * *$ & $34.16^{* *}$ & $34.16^{* * *}$ & $38.99 * *$ \\
\hline 2 & -0.03 & -0.78 & $-57.28 * *$ & $-57.28 * *$ & $-53.37 * *$ \\
\hline 3 & -0.03 & $-1.11 *$ & $23.11 * *$ & $23.11 * *$ & $14.38 * *$ \\
\hline LSD 0.05 & 0.06 & 0.96 & 6.45 & 6.45 & 5.66 \\
\hline LSD 0.01 & 0.08 & 1.27 & 8.54 & 8.54 & 7.50 \\
\hline \multicolumn{6}{|l|}{ Lines } \\
\hline 1 & $1.07 * *$ & -1.67 & $62.14 * *$ & $62.14 * *$ & $41.90 * *$ \\
\hline 2 & -0.49 & -0.01 & $-77.98 * *$ & $-77.98 * *$ & $-43.33 * *$ \\
\hline 3 & 0.40 & 1.44 & $90.01 * *$ & $90.01 * *$ & $71.90 * *$ \\
\hline 4 & $-0.93^{*}$ & -0.90 & 12.80 & 12.80 & $28.56^{* *}$ \\
\hline 5 & -0.04 & -0.23 & $50.80 * *$ & $50.80 * *$ & -1.66 \\
\hline 6 & 0.40 & -0.78 & -7.86 & -7.86 & $-19.44 * *$ \\
\hline 7 & 0.18 & $3.33 * *$ & $34.24 * *$ & $34.24 * *$ & $22.79 * *$ \\
\hline 8 & -0.04 & -1.23 & $38.25 * *$ & $38.25 * *$ & $30.67 * *$ \\
\hline 9 & 0.40 & -1.01 & $-46.42 * *$ & $-46.42 * *$ & $-49.10 * *$ \\
\hline 10 & 0.18 & -1.12 & $33.13 * *$ & $33.13 * *$ & $37.67 * *$ \\
\hline 11 & -0.04 & -0.23 & $64.90 * *$ & $64.90 * *$ & $58.12 * *$ \\
\hline 12 & -0.49 & 2.22 & $-56.54 * *$ & $-56.54 * *$ & $-47.99 * *$ \\
\hline 13 & 0.63 & -1.56 & $-81.42 * *$ & $-81.42 * *$ & $-58.99 * *$ \\
\hline 14 & -0.71 & 1.33 & $-124.99 * *$ & $-124.99 * *$ & $-84.66^{* *}$ \\
\hline 15 & $0.85^{*}$ & -1.90 & -1.42 & -1.42 & -3.10 \\
\hline 16 & $-1.38 * *$ & $2.33 *$ & 10.36 & 10.36 & $16.67^{*}$ \\
\hline LSD 0.05 & 0.72 & 2.22 & 14.90 & 14.90 & 13.07 \\
\hline LSD 0.01 & 0.95 & 2.94 & 19.73 & 19.73 & 17.31 \\
\hline
\end{tabular}

* and ${ }^{* *}$ indicate significance at 0.05 and 0.01 levels of probability, respectively.

Specific combining ability effects:

Specific combining ability effects ( $\mathrm{Sij}$ ) of the top crosses for all traits are presented in (table 4).

Significant and negative $\mathrm{Sij}$ effects for days to $50 \%$ tasseling were detected by top crosses L3xT3, L1xT2 and L11xT1. These crosses expressed SCA effects reached $-2.26,-1.40$ and -1.23 for the mention top crosses, respectively.

For days to $50 \%$ silking date the top cross L3xT3 exhibited high significant and negative (Sij) effects. Early maturity crosses in maize is convenient for escaping destructive injuries caused by borer like
Sesamia cretica, Chilo simplex and Pyrausta nubilialis. Similar results were reported by El-Hosary and El-Fiki (2015) and Ismail (2019 a \& b).

As for plant height, five top crosses L2xT2, L5xT3, L7xT2, L12xT1 and L14xT1 exhibited negative and significant SCA effects. Meanwhile, the top crosses L7xT1 and L12xT2 showed high positive and significant SCA effects for this trait. Meanwhile, the other top crosses showed insignificant SCA effects. As for ear height, the high significant and negative SCA effects were detected by the top crosses L1xT2, L3xT2, L2xT2, L5xT3, 
L13xT3 and L14xT1. In this respect, Zambezi et al ,(1986) pointed out that inbred tester are preferred over broad base testers and mentioned two practical response for preferring inbred line (narrow base) testers to broad-base testers. First sampling errors are likely to occur with hetero genous testers. Second, use of an inbred line or single cross tester may permit quicker utilization of new lines in commercial hybrids, especially if the testers already in commercial use. As for No of kernels/ row, the top crosses L2xT3, L12xT2 and L15xT1 exhibited high significant and positive SCA effects. For No of rows/ ear, the top crosses L3xT1, L6xT1, L4xT2, L8xT3 and L11xT3 expressed significantly positive $\mathrm{Sij}$ effects. Regarding ear weight/ plant, the most desirable SCA effects were exhibited in the top crosses combination between $\mathrm{T} 1$ and each of L1, L2, L4, L7, L9, L10, L11 and L13. Also, between T2 and each of L5, L6, L8, L9, L10, L12, L15 and L16, and between T3 and each of L4, L5, L8, L12, L13 and L14. The most desirable SCA effects for grain yield/ plant were detected by the top crosses combinations between T1 and each of L1,L2,L4,L7,L9, L10,L11, L13 and L16, also, between T2 and each of L3,L6, L8, L9,L10,L12,L15 and L16,also between T3 and each of L4,L5,L8,L12 and L14.

For shelling\%, the top cross L3xT2 exhibited significantly positive $\mathrm{Sij}$ effects. These test-crosses might be of interest in breeding programs as most of them involved at least one good combiner for the concerned traits. These test crosses could be of interest to obtain synthetic varieties or produced inbred lines.

It could be concluded that testers of broad genetic base are more efficient than those of the narrow genetic base for evaluation of GCA inbred lines of maize.

Table 4. Specific combining ability effects for all top crosses

\begin{tabular}{|c|c|c|c|c|c|c|c|c|c|}
\hline \multirow[b]{2}{*}{$\begin{array}{l}\text { Trait } \\
\text { Tester } \\
\text { Line }\end{array}$} & \multicolumn{3}{|c|}{ Tasseling date (Days to $50 \%$ shed) } & \multicolumn{3}{|c|}{ Silking date (Days to $50 \%$ silk) } & \multicolumn{3}{|c|}{ Plant height $(\mathrm{cm})$} \\
\hline & $\begin{array}{c}\text { T1 } \\
\text { Inbred line } \\
\text { L.921 }\end{array}$ & $\begin{array}{c}\text { T2 } \\
\text { Pop.(I } \\
y- \\
236)\end{array}$ & $\begin{array}{c}\text { T3 } \\
\text { SC. }(G \\
\text { old } 21)\end{array}$ & $\begin{array}{c}\text { T1 } \\
\text { Inbred line } \\
\text { L.921 }\end{array}$ & $\begin{array}{c}\text { T2 } \\
\text { Pop.(I } \\
y- \\
236)\end{array}$ & $\begin{array}{c}\text { T3 } \\
\text { SC. }(G \\
\text { old } 21)\end{array}$ & $\begin{array}{c}\text { T1 } \\
\text { Inbre } \\
\text { d line } \\
\text { L.92 } \\
1\end{array}$ & $\begin{array}{c}\text { T2 } \\
\text { Pop.(I } \\
y- \\
236)\end{array}$ & $\begin{array}{c}\text { T3 } \\
\text { SC. (G } \\
\text { old 21) }\end{array}$ \\
\hline 1 & 0.99 & $-1.40^{*}$ & 0.41 & 0.93 & -1.36 & 0.43 & 0.25 & -8.66 & 8.41 \\
\hline 2 & -0.23 & -0.29 & 0.52 & -0.18 & -0.14 & 0.32 & 9.88 & $\overline{-}^{-} 6^{*}$ & 6.98 \\
\hline 3 & $1.99 * *$ & 0.26 & $-2.26^{* *}$ & $2.04^{* *}$ & 0.08 & $-2.13^{* *}$ & 11.40 & -11.97 & 0.57 \\
\hline 4 & 0.77 & -0.29 & -0.48 & 0.82 & -0.47 & -0.35 & -2.73 & 6.70 & -3.97 \\
\hline 5 & -0.67 & 0.93 & -0.26 & -1.29 & 1.08 & 0.21 & -0.14 & $15.42^{*}$ & $\begin{array}{l}- \\
15.28 *\end{array}$ \\
\hline 6 & -0.23 & -0.63 & 0.85 & -0.18 & -0.81 & 0.99 & -3.68 & -3.65 & 7.32 \\
\hline 7 & 0.22 & -1.18 & 0.97 & 0.37 & -1.25 & 0.87 & $\underset{*}{15.45}$ & ${ }^{-}$ & 2.48 \\
\hline 8 & -0.90 & 0.71 & 0.19 & -0.74 & 0.64 & 0.10 & -6.98 & -0.88 & 7.86 \\
\hline 9 & -0.67 & $1.26^{*}$ & -0.59 & -0.51 & 1.19 & -0.68 & $1.89^{*}$ & -2.51 & 0.62 \\
\hline 10 & -0.45 & 0.15 & 0.30 & 0.04 & 0.08 & -0.13 & 13.56 & -13.08 & -0.48 \\
\hline 11 & $-1.23^{*}$ & 0.71 & 0.52 & -1.29 & 1.08 & 0.21 & $\begin{array}{l}0.43 \\
-\end{array}$ & 12.06 & -12.50 \\
\hline 12 & -0.23 & 1.04 & -0.81 & -0.18 & 0.86 & -0.68 & 19.53 & $15.43^{*}$ & 4.10 \\
\hline 13 & -0.78 & 0.15 & 0.63 & -1.07 & 0.31 & 0.76 & $\begin{array}{c}12.28 \\
-\end{array}$ & -1.62 & -10.65 \\
\hline 14 & 0.22 & -0.51 & 0.30 & 0.15 & -0.47 & 0.32 & $\underset{*}{17.02}$ & 6.87 & 10.15 \\
\hline 15 & 0.55 & -0.51 & -0.03 & 0.60 & -0.36 & -0.24 & -6.89 & 13.24 & -6.35 \\
\hline 16 & 0.66 & -0.40 & -0.26 & 0.49 & -0.47 & -0.01 & -8.17 & 7.43 & 0.73 \\
\hline $\begin{array}{l}\text { LSD sij } \\
0.05\end{array}$ & & .21 & & & 1.37 & & & 14.19 & \\
\hline $\begin{array}{l}\text { LSD sij } \\
0.01\end{array}$ & & .60 & & & 1.82 & & & 18.80 & \\
\hline $\begin{array}{l}\text { LSD sij-skl } \\
0.05\end{array}$ & & .71 & & & 1.94 & & & 20.07 & \\
\hline
\end{tabular}

\footnotetext{
${ }^{*}$ and ${ }^{* *}$ indicate significance at 0.05 and 0.01 levels of probability, respectively.
} 


\begin{tabular}{|c|c|c|c|c|c|c|c|c|c|}
\hline \multirow[b]{2}{*}{$\begin{array}{c}\text { Trait } \\
\text { Tester } \\
\text { Line }\end{array}$} & \multicolumn{3}{|c|}{ Ear height $(\mathrm{cm})$} & \multicolumn{3}{|c|}{ Number of rows $\backslash$ ear } & \multicolumn{3}{|c|}{ 100-kernel weight $(\mathrm{g})$} \\
\hline & $\begin{array}{c}\mathrm{T} 1 \\
\text { Inbred line } \\
\text { L.921 }\end{array}$ & $\begin{array}{c}\text { T2 } \\
\text { Pop.( } \\
\text { Iy- } \\
236)\end{array}$ & $\begin{array}{c}\text { T3 } \\
\text { SC. }(\mathrm{G} \\
\text { old } \\
21) \\
\end{array}$ & $\begin{array}{c}\mathrm{T} 1 \\
\text { Inbred line } \\
\text { L.921 } \\
\end{array}$ & $\begin{array}{c}\text { T2 } \\
\text { Pop.( } \\
\text { Iy- } \\
236)\end{array}$ & $\begin{array}{c}\text { T3 } \\
\text { SC. }(\mathrm{G} \\
\text { old } \\
21) \\
\end{array}$ & $\begin{array}{c}\mathrm{T} 1 \\
\text { Inbred } \\
\text { line } \\
\text { L.921 } \\
\end{array}$ & $\begin{array}{c}\text { T2 } \\
\text { Pop.(Iy- } \\
\text { 236) }\end{array}$ & $\begin{array}{c}\text { T3 } \\
\text { SC. }(\mathrm{G} \\
\text { old } \\
21) \\
\end{array}$ \\
\hline 1 & -1.85 & $\begin{array}{c}- \\
13.93 \\
* *\end{array}$ & $\begin{array}{c}15.77 * \\
*\end{array}$ & $-1.61 *$ & 0.47 & 1.14 & 1.44 & 0.78 & -2.22 \\
\hline 2 & 5.00 & $\begin{array}{c}- \\
13.18 \\
* *\end{array}$ & 8.18 & 0.61 & -0.64 & 0.03 & 0.44 & 3.11 & -3.56 \\
\hline 3 & 8.89 & $\begin{array}{c}- \\
11.52 \\
*\end{array}$ & 2.64 & $1.72 * *$ & -0.86 & -0.86 & 1.33 & 0.33 & -1.67 \\
\hline 4 & -4.17 & -2.48 & 6.65 & 0.39 & -0.19 & -0.19 & 2.67 & $-6.33 * *$ & 3.67 \\
\hline 5 & -0.72 & $\begin{array}{c}13.30 \\
* *\end{array}$ & $\begin{array}{c}- \\
12.57 * \\
*\end{array}$ & -1.17 & $1.58 *$ & -0.42 & -2.67 & -2.00 & $4.67 *$ \\
\hline 6 & 8.13 & -6.68 & -1.45 & $2.39 * *$ & -0.86 & $-1.53^{*}$ & -2.44 & -1.11 & 3.56 \\
\hline 7 & 3.74 & -1.77 & -1.97 & -0.72 & -0.64 & $1.36^{*}$ & 0.78 & 2.78 & -3.56 \\
\hline 8 & -9.12 & 2.23 & 6.89 & 0.17 & $\begin{array}{c}- \\
1.75^{*} \\
*\end{array}$ & $1.58^{*}$ & -2.00 & 3.00 & -1.00 \\
\hline 9 & 1.28 & 3.13 & -4.41 & $-1.61^{*}$ & $\begin{array}{c}3.14^{*} \\
*\end{array}$ & $-1.53^{*}$ & $6.11 * *$ & $-5.22 * *$ & -0.89 \\
\hline 10 & 7.63 & -5.61 & -2.02 & 0.61 & 0.03 & -0.64 & 1.22 & -1.11 & -0.11 \\
\hline 11 & -2.59 & -3.00 & 5.59 & -0.50 & -1.08 & $1.58^{*}$ & -3.33 & $4.67 *$ & -1.33 \\
\hline 12 & -2.75 & 5.74 & -3.00 & -0.06 & 0.69 & -0.64 & -3.44 & 1.22 & 2.22 \\
\hline 13 & 3.74 & 5.56 & - & 0.17 & -1.08 & 0.92 & -0.33 & 1.33 & -1.00 \\
\hline 14 & $-15.19 * *$ & $\begin{array}{c}19.40 \\
* *\end{array}$ & -4.21 & 0.17 & 0.25 & -0.42 & -0.56 & $-3.89 *$ & $4.44 *$ \\
\hline 15 & 0.20 & -0.78 & 0.58 & $-1.39 *$ & 0.69 & 0.69 & 2.33 & -0.33 & -2.00 \\
\hline 16 & -2.22 & $9.60 *$ & -7.37 & 0.83 & 0.25 & -1.08 & -1.56 & 2.78 & -1.22 \\
\hline $\begin{array}{c}\text { LSD sij } \\
0.05\end{array}$ & & 9.26 & & & 1.25 & & & 3.84 & \\
\hline $\begin{array}{c}\text { LSD sij } \\
0.01\end{array}$ & & 12.27 & & & 1.65 & & & 5.09 & \\
\hline $\begin{array}{c}\text { LSD sij-skl } \\
0.05\end{array}$ & & 13.10 & & & 1.76 & & & 5.43 & \\
\hline
\end{tabular}

${ }^{*}$ and ${ }^{* *}$ indicate significance at 0.05 and 0.01 levels of probability, respectively. 
Table (4) : Cont.

\begin{tabular}{|c|c|c|c|c|c|c|c|c|c|}
\hline Trait & Ear we & ght / plant & & Grain & yield/ plat & & & Shelling & \\
\hline $\begin{array}{l}\text { Tester } \\
\text { Line }\end{array}$ & $\begin{array}{c}\text { T1 } \\
\text { Inbred line } \\
\text { L.921 }\end{array}$ & $\begin{array}{c}\text { T2 } \\
\text { Pop.(I } \\
\text { y-236) }\end{array}$ & $\begin{array}{c}\text { T3 } \\
\text { SC. (G } \\
\text { old 21) }\end{array}$ & $\begin{array}{c}\text { T1 } \\
\text { Inbred line } \\
\text { L.921 }\end{array}$ & $\begin{array}{c}\text { T2 } \\
\text { Pop.(I } \\
\text { y- } \\
236)\end{array}$ & $\begin{array}{c}\text { T3 } \\
\text { SC.(G } \\
\text { old 21) }\end{array}$ & $\begin{array}{c}\text { T1 } \\
\text { Inbr } \\
\text { ed } \\
\text { line } \\
\text { L. } 92 \\
1 \\
\end{array}$ & $\begin{array}{c}\text { T2 } \\
\text { Pop.(I } \\
\text { y- } \\
236)\end{array}$ & $\begin{array}{c}\text { T3 } \\
\text { SC. }(\mathrm{G} \\
\text { old } 21)\end{array}$ \\
\hline 1 & $49.28 * *$ & -14.61 & $\begin{array}{c}- \\
34.76 * \\
*\end{array}$ & $10.49 * *$ & 1.10 & $\begin{array}{c}- \\
11.59 * \\
*\end{array}$ & 2.48 & 0.29 & -2.77 \\
\hline 2 & $111.39 * *$ & $\begin{array}{c}- \\
115.83 \\
* *\end{array}$ & 4.44 & $20.80 * *$ & $\begin{array}{c}- \\
22.86 \\
* *\end{array}$ & 2.06 & $\begin{array}{c}- \\
1.97\end{array}$ & -0.49 & 2.45 \\
\hline 3 & $-34.93 * *$ & 22.18 & 12.75 & $-7.71 * *$ & $\begin{array}{c}14.96 \\
* *\end{array}$ & $\begin{array}{c}- \\
7.25 * *\end{array}$ & $\begin{array}{c}- \\
1.41\end{array}$ & $\begin{array}{c}4.74 * \\
*\end{array}$ & $-3.33 *$ \\
\hline 4 & $57.28 * *$ & $\begin{array}{c}- \\
90.94 * \\
*\end{array}$ & $33.66 *$ & $9.49 * *$ & $\begin{array}{c}- \\
17.50 \\
* *\end{array}$ & $8.01 * *$ & 1.30 & -0.49 & 1.78 \\
\hline 5 & $-119,72 * *$ & $\begin{array}{c}48.06 * \\
*\end{array}$ & $\begin{array}{c}71.66^{*} \\
*\end{array}$ & $-15.40 * *$ & $5 . .06^{*}$ & $\begin{array}{c}20.46^{*} \\
*\end{array}$ & 0.03 & -1.49 & 1.45 \\
\hline 6 & 20.61 & $33.39 *$ & $\begin{array}{c}- \\
54.00 * \\
*\end{array}$ & 1.62 & $4.56^{*}$ & $6.19^{* *}$ & 1.63 & -0.15 & 1.78 \\
\hline 7 & $162.51 * *$ & $\begin{array}{c}- \\
103.08 \\
* *\end{array}$ & $\begin{array}{c}- \\
59.44^{*} \\
*\end{array}$ & $21.18 * *$ & $\begin{array}{c}- \\
11.08 \\
* *\end{array}$ & $\begin{array}{c}- \\
10.10^{*}\end{array}$ & $\begin{array}{c}- \\
1.74\end{array}$ & 2.07 & -0.33 \\
\hline 8 & $-111.16^{* *}$ & $\begin{array}{l}40.94 * \\
*\end{array}$ & $\begin{array}{c}70.22 * \\
*\end{array}$ & $-20.68 * *$ & $\begin{array}{c}11.54 \\
* *\end{array}$ & $9.32 * *$ & $\overline{-}-$ & 1.74 & -1.33 \\
\hline 9 & $44.84 * *$ & $\begin{array}{l}93.94 * \\
*\end{array}$ & $\begin{array}{c}- \\
138.78 \\
* *\end{array}$ & $7.89 * *$ & $\begin{array}{c}11.83 \\
* *\end{array}$ & $\begin{array}{c}- \\
19.72 * \\
*\end{array}$ & 0.59 & -2.26 & 1.67 \\
\hline 10 & $82.96 * *$ & $\begin{array}{c}39.40^{*} \\
*\end{array}$ & $\begin{array}{c}- \\
122.36 \\
* *\end{array}$ & $12.14 * *$ & $4.87 *$ & $\begin{array}{c}- \\
17.01 * \\
*\end{array}$ & 0.41 & -1.26 & 1.67 \\
\hline 11 & $31.18 *$ & $29.04 *$ & -2.14 & $9.58 * *$ & $\begin{array}{c}- \\
9.75^{*} \\
*\end{array}$ & 0.17 & 2.59 & -2.93 & 0.34 \\
\hline 12 & $-296.04 * *$ & $\begin{array}{c}154.73 \\
* *\end{array}$ & $\begin{array}{c}141.31 \\
* *\end{array}$ & $-51.40 * *$ & $\begin{array}{c}23.41 \\
* *\end{array}$ & $\begin{array}{c}27.99 * \\
*\end{array}$ & 0.37 & -1.82 & 1.45 \\
\hline 13 & $59.84 * *$ & $\begin{array}{c}- \\
88.42 * \\
*\end{array}$ & $28.59^{*}$ & $8.80 * *$ & $\begin{array}{c}- \\
11.73 \\
* *\end{array}$ & 2.92 & 0.19 & 0.96 & -0.77 \\
\hline 14 & $-88.60 * *$ & $\begin{array}{c}- \\
88.16^{*}\end{array}$ & $\begin{array}{c}176.75 \\
* *\end{array}$ & $-16.80 * *$ & $\begin{array}{c}- \\
8.59 * \\
*\end{array}$ & $\begin{array}{c}25.39 * \\
*\end{array}$ & 0.19 & 2.96 & -2.77 \\
\hline 15 & 8.17 & $\begin{array}{l}52.61 * \\
*\end{array}$ & $\begin{array}{c}- \\
60.78^{*} \\
*\end{array}$ & 3.49 & $\begin{array}{c}7.30 * \\
*\end{array}$ & $\begin{array}{c}- \\
10.79 * \\
*\end{array}$ & 1.59 & -1.26 & -0.33 \\
\hline 16 & 22.39 & $44.83 * *$ & $-67.22 * *$ & $6.67 * *$ & $7.01 * *$ & $\begin{array}{c}- \\
13.68 * *\end{array}$ & 1.59 & -0.60 & -0.99 \\
\hline $\begin{array}{c}\text { LSD sij } \\
0.05\end{array}$ & \multicolumn{3}{|c|}{25.81} & \multicolumn{3}{|c|}{4.53} & \multicolumn{3}{|c|}{3.21} \\
\hline $\begin{array}{c}\text { LSD sij } \\
0.01\end{array}$ & \multicolumn{3}{|c|}{34.18} & \multicolumn{3}{|c|}{6.00} & \multicolumn{3}{|c|}{4.25} \\
\hline $\begin{array}{c}\text { LSD sij-skl } \\
0.05\end{array}$ & \multicolumn{3}{|c|}{36.50} & \multicolumn{3}{|c|}{6.41} & \multicolumn{3}{|c|}{4.53} \\
\hline
\end{tabular}

${ }^{*}$ and ${ }^{* *}$ indicate significance at 0.05 and 0.01 levels of probability, respectively. 


\section{References}

Abd El-Zaher, I. N. (2016). Line x tester mating design for estimation combining ability in maize. Assiut J . Agri. Sci., 47(4):16-31.

Amer, E.A. and A.A. El-Shenawy (2007) Combining ability for new twenty yellow maize inbred lines. J. Agric. Sci. 32(9):7053-7062.

Ahmed, M.H. Eatemad (2008). combining ability for yield and its attributes in newly developed yellow maize indred lines . Assiut J . Agri. Sci ., 39(5): 1-15.

Ahmed, S.; S. Begum; A. Islam; M. Ratna and R. Karim (2017) Combining ability estimates in maize (Zea Mays L.) through line $\mathrm{x}$ tester analysis. Bangladesh J. of Agric. Res., 42(3): 425-436.

Arunkumar, B.; E. Gangapp; S. Ramesh; L. D. Savithramma; N. Nagaraju and R. Lokesha (2020) Stability analysis of maize (Zea mays L.) hybrids for grain yield and its attributing traits using Eberhart and Russel model. Current J. of App. Sci. and Tech., 39(1): 52-63.

Bayoumi, R. A.; S.M. Shoker; G.Y. Hamam; A.A.A. El-Hosary (2018) Determination of combining ability for some new yellow maize inbred lines using line $\mathrm{x}$ tester model. Annals of Agric. Sci., Moshtohor. 56(2): 305-316.

Darshan, S. S. and S. Marker (2019). Heterosis and combining ability for grain yield and its component characters in quality protein maize (Zea mays L.) hybrids . Electronic J . Plant Breed., 10 (1): 111-118.

El-Morshidy, M.A.; E.A. Hassaballa; Sh.F. AbouElsaad and M.A. Abd El-Moula (2003) Combining ability and type of gene action in maize under favorable and water stress environments. Proc. Plant Breed. Conf. Giza Egypt, 26: 55-57.

EL-Hosary A.A.A. and A. A. El-Gammaal (2013). Utilization of line $\mathrm{x}$ tester model for evaluating the combining ability of some new white maize inbred lines. Egypt. J. Plant Breed. 17(1):79-72.

El-Hosary, A.A.A. (2014) Relative values of three different testers in evaluating combining ability of new maize inbred lines. Intern. J. of Plant Breed. and Genetics. 5(2): 57-65.

El-Hosary, A.A.A. and I.A.I. El-Fiki (2015) Diallel cross analysis for earliness, yield, its components and resistance to late wilt in maize. Intern. J. of Agric. Sci. and Res. (IJASR), 5(6): 199-210.

El-Hosary, A.A.A. (2020) Diallel analysis of some quantitative traits in eight inbred lines of maize and GGE biplot analysis for elite hybrids. $\mathbf{J}$ of Plant Production, Mansoura Univ., Vol. 11 (3): $275-283$.

Grogan, C.O. and M.S. Zuber (1957) A comparative study of top cross testers parents of maize. Agron. J. 49:68-72.

Gamea, H. A. A. (2019) . Genetic analysis for grain yield and some agronomic traits in some new white maize inbred lines by using line $\mathrm{x}$ tester analysis . Alex . J. Agri. Sci., 64(5):309-317.

Hosna, G. C.; S. Alamerew; B. Tadesse and T. Menamo (2015). Test cross performance and combining ability of maize (Zea mays L.) inbred lines at Bako, Western Ethiopia . Global J . Sci. Res., 15 (4): 1-24

Ibrahim, M.H.A. and M.M.A. Osman (2005) : Combining ability estimates and type of gene action for white maize (Zea mays L.) top crosses. Egypt. J. Appl. Sci., 20 (10B): 483-500

Iqbal, A. M; F. A. Nehvi; S. A. Wani; R. Qadir and Z. A. Dar (2007). Combining ability analysis for yield and yield related traits in maize (Zea mays L.). Int. J. Plant Breed. Gene., 1(2):101-105.

Ismail, M.R.; A.A. El-Hosary; M.E.M. ElBadawy; T.A.E. Abdallah (2019 a) Diallel analysis for yield and component traits in maize (Zea mays) under infestation and non-infestation with pink stem borer (Sesamia cretica). Indian J. of Agric. Sci., 89 (11): 1953-1958.

Ismail, M.R.; A.A. El-Hosary; M.E.M. ElBadawy; T.A.E. Abdallah (2019 b) Combining ability studies on resistance to pink stem borer (Sesamia cretica) in new yellow maize (Zea mays) hybrids. Electronic Journal of Plant Breeding, 10 (4): 1376-1382.

Ismail, M.R.; A.A. El-Hosary; M.E.M. El-Badawy (2020) Comparison among three different testers for the evaluation of new maize inbred lines (Zea mays L.). Maydica, 65(1):1-6.

Kempthorne O. (1957) An introduction to genetic statistics. John-Wiley and Sons Inc. Landon, UK.

Lai, M.; D. Singh and S. Dass (2011). General and specific combining ability studies in maize using line $x$ tester design. Agri. Sci. Dige., 31(1):8-13

Matzinger, D.F. (1953). Comparison of three types of testers for the evaluation of inbred lines of corn. Agron. J. 45:493-495.

Osman, M.M.A and M.H.A. Ibrahim (2007). A study on combining ability of new yellow maize inbred lines using line $\mathrm{x}$ tester analysis . J. Agric. Sci., Mansoura Uni., 8:815-830.

Mbuvi, B.; M. Mwimali and M. Githiri (2018). Estimation of general and specific combining ability of maize inbred lines using single cross testers for earliness . World J. Agri. Res., 6 (2):37-48.

Rajitha, A.; D. R. Babu; L. M. Ahmed and V. S. Rao (2014). Heterosis and combining ability for grain yield and yield component traits in maize (Zea mays L.). Electronic J . Plant breed.; 5(3): 378-384.

Sprague, G.F. (1939) An estimation of the number of top crossed plants required for adequate representation of corn variety. J. Am. Soc. Agron. 38:11-16. 
Sadek, S.E.; M.S.M. Soliman; A.A.Barakat and K.I. Khalifa (2002) Top-crosses analysis for selecting maize lines in the early self generations. Menoufia J. Agric. Res. 27(2): 197-213.

Sowmya, H.H.; M.Y. Kamatar; G. Shanthakumar; S.M. Brunda; T.V. Shadakshari; B.M.; S. Babu; R.S. Singh (2018) Stability analysis of maize hybrids using Eberhart and Russel Model. Intern. J. of Current Micr. and App. Sci.,7 (2):3336-3343.

Zambezi, B .T.;E.S. Horner and F.G. Martin (1986): Inbred lines as testers for general combining ability in maize . Crop Sci. 26: 908 910.

التحليل الوراثى للصفات الهامة فى هجن الذرة الثامية الصفراء الجديدة باستخدام موديل السلالة x الكشاف

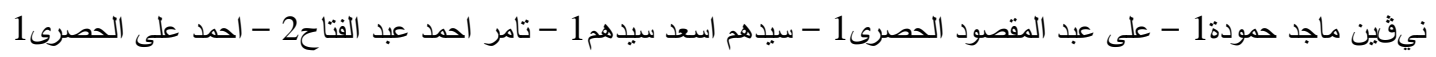

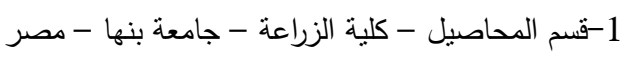

2- قسم بحوث الذرة الثامية - معهد بحوث المحاصيل الحقلية - مركز البحوث الزراعية

أجري التهجين بين ستة عشر سلالة مرباه داخليا و ثلاث كثافات من الذرة الثامية لنقلقير القدرة على الثآلف لطول النبات , إرتفاع

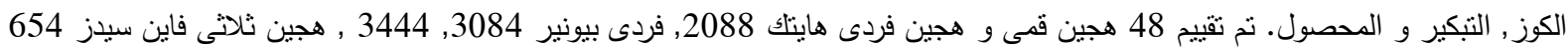

وهجين ثلاثى 352 بمزرعة البحوث و التجارب - كلية الزراعة بمشتهر - جامعة بنها فى تصميم قطاعات كاملة العشوائية بثلاث مكررات.

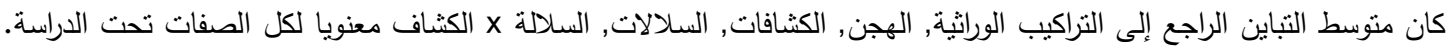

أظهر التباين الغير مضيف دورا أكثر أهمية فى وراثة الصفات محل الدراسة. و بالنسبة لمساهمه السلالات فى السلالة x الكثاف كان اكثر من

مساهمة الكثافات تحت الدراسه. أظهرت السلالة رقم 11 قدرة عامة على الثآلف مرغوبة لصفات التبكير و أظهرت السلالده رقم 3 و تبعها 11 و و 1 و 10معنوية موجبة للقدرة الخاصه على التآلف لصفة محصول الحبوب / نبات. أظهر الهجين L3XT3 قدرة خاصة على التآلف سالبة و

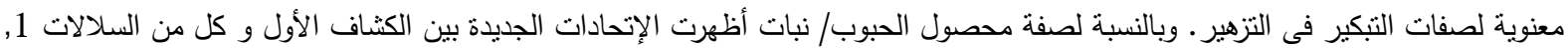
2, 4, 7, 9, 10, 11 , 13 و 16 و أيضا بين الكثاف الثانى و السلالات 3, 6 , 8, 9, 10, 12, 15 و و 16 و الإتحادات بين الكثاف

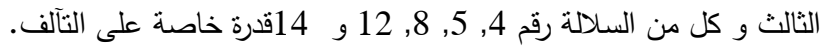

